\title{
Cosmological parameters and neutrino masses from the final Planck and full-shape BOSS data
}

\author{
Mikhail M. Ivanov $\oplus^{1,2, *}$ Marko Simonović $\odot,{ }^{3, \dagger}$ and Matias Zaldarriaga ${ }^{4,+}$ \\ ${ }^{1}$ Center for Cosmology and Particle Physics, Department of Physics, New York University, \\ New York, New York 10003, USA \\ ${ }^{2}$ Institute for Nuclear Research of the Russian Academy of Sciences, \\ 60th October Anniversary Prospect, 7a, 117312 Moscow, Russia \\ ${ }^{3}$ Theoretical Physics Department, CERN, 1 Esplanade des Particules, Geneva 23, CH-1211, Switzerland \\ ${ }^{4}$ School of Natural Sciences, Institute for Advanced Study, \\ 1 Einstein Drive, Princeton, New Jersey 08540, USA
}

(Received 26 December 2019; accepted 9 March 2020; published 3 April 2020)

\begin{abstract}
We present a joint analysis of the Planck cosmic microwave background (CMB) and Baryon Oscillation Spectroscopic Survey (BOSS) final data releases. A key novelty of our study is the use of a new full-shape (FS) likelihood for the redshift-space galaxy power spectrum of the BOSS data, based on an improved perturbation theory template. We show that the addition of the redshift-space galaxy clustering measurements breaks degeneracies present in the CMB data alone and tightens constraints on cosmological parameters. Assuming the minimal $\Lambda \mathrm{CDM}$ cosmology with massive neutrinos, we find the following lateUniverse parameters: the Hubble constant $H_{0}=67.95_{-0.52}^{+0.66} \mathrm{~km} \mathrm{~s}^{-1} \mathrm{Mpc}^{-1}$, the matter density fraction $\Omega_{m}=0.3079_{-0.0085}^{+0.0065}$, the mass fluctuation amplitude $\sigma_{8}=0.8087_{-0.0072}^{+0.012}$, and an upper limit on the sum of neutrino masses $M_{\text {tot }}<0.16 \mathrm{eV}$ (95\% C.L.). This can be contrasted with the Planck-only measurements: $H_{0}=67.14_{-0.72}^{+1.3} \mathrm{~km} \mathrm{~s}^{-1} \mathrm{Mpc}^{-1}, \Omega_{m}=0.3188_{-0.016}^{+0.0091}, \sigma_{8}=0.8053_{-0.0091}^{+0.019}$, and $M_{\text {tot }}<0.26 \mathrm{eV}$ (95\% C.L.). Our bound on the sum of neutrino masses relaxes once the hierarchy-dependent priors from the oscillation experiments are imposed. The addition of the new FS likelihood also constrains the effective number of extra relativistic degrees of freedom, $N_{\text {eff }}=2.88 \pm 0.17$. Our study shows that the current FS and the pure baryon acoustic oscillation data add a similar amount of information in combination with the Planck likelihood. We argue that this is just a coincidence given the BOSS volume and efficiency of the current reconstruction algorithms. In the era of future surveys FS will play a dominant role in cosmological parameter measurements.
\end{abstract}

DOI: 10.1103/PhysRevD.101.083504

\section{INTRODUCTION}

Planck cosmic microwave background (CMB) data have been the leading cosmological probe with unprecedented measurement of cosmological parameters [1]. As powerful as they are, the CMB data possess some internal parameter degeneracies, which compromise the accuracy of cosmological constraints, especially for nonminimal extensions of the base $\Lambda \mathrm{CDM}$ model. A way to break some of these degeneracies is to use additional information form the

\footnotetext{
*mi1271@nyu.edu

marko.simonovic@cern.ch

*matiasz@ias.edu
}

Published by the American Physical Society under the terms of the Creative Commons Attribution 4.0 International license. Further distribution of this work must maintain attribution to the author(s) and the published article's title, journal citation, and DOI. large-scale structure (LSS) surveys. The most well-known example is a combination of the Planck likelihood with the geometric location of the baryon acoustic oscillation (BAO) peak inferred from the galaxy correlation function [2]. There are two major reasons why this combination is often employed. First, the BAO peak is relatively easy to measure and it is very robust against various possible systematic effects of spectroscopic galaxy surveys. Second, the reconstruction algorithms used to "sharpen" the BAO feature exploit higher $n$-point correlation functions of the nonlinear density field, which significantly improves the measurement of the location of the BAO peak [3-6]. This allows for an accurate and robust measurement of the BAO scale, which in turn breaks degeneracies of the Planck likelihood [1,2].

One important example where the BAO information plays a notable role is the constraint on the sum of neutrino masses $M_{\text {tot }}$. Significant efforts from both particle physics and cosmology confined this parameter to the narrow range 


$$
0.06 \mathrm{eV}<M_{\text {tot }}<0.12 \mathrm{eV}
$$

where the upper bound is a 95\% confidence interval from observations of temperature and polarization fluctuations in the $\mathrm{CMB}$ along with $\mathrm{BAO}$ in the distribution of different tracers of matter [1], whereas the lower limit is given by the flavor oscillation experiments ${ }^{1}$ [7]. Remarkably, the combination of CMB and LSS data (e.g., Refs. [2,8-13]; see also Ref. [14] and references therein) gives a much tighter upper bound on the total neutrino mass than laboratory experiments like KATRIN [15].

While this and other similar examples show the importance of combining the $\mathrm{BAO}$ data with the $\mathrm{CMB}$ observations, the position of the $\mathrm{BAO}$ peak (including reconstruction) represents only a part of the cosmological information encoded in the clustering pattern of galaxies in redshift space. Complementary information is in the broadband of the power spectrum (as well as higher $n$-point functions). This information is naturally extracted using the full-shape (FS) analysis. In this approach the whole power spectrum is exploited and, unlike the BAO measurement alone, all cosmological parameters can be constrained independently of the CMB data [16-19]. Remarkably, the FS information allows one to measure the late-time matter density fraction $\Omega_{m}$ to $3 \%$ and the Hubble parameter $H_{0}$ to $2 \%$ precision without the shape and sound horizon priors from the CMB. These measurements are not possible with the BAO and full-shape studies that are based on scaling parameters (see Refs. [2,20]). The first goal of this paper is to combine the new FS likelihood of the BOSS data presented in Ref. [16] with the Planck CMB likelihood and measure the cosmological parameters.

Having BAO and FS analyses at hand, one immediate question to ask is how they compare. It is hard to give a simple and intuitive answer for several reasons. On the one hand, the reconstructed $\mathrm{BAO}$ feature is sharper, but the broadband can be measured much better (the amplitude of the $\mathrm{BAO}$ wiggles is a few percent of the broadband). On the other hand, the broadband has no strong features and its shape is uncertain due to the nonlinear evolution and instrumental systematic effects. However, the FS analysis does include the damped $\mathrm{BAO}$ wiggles, which still contain a significant amount of information. Given all of these differences, the second goal of this paper is to answer the following simple questions: (a) How do the cosmological parameter measurements compare between the BAO and the FS analyses of the BOSS data in combination with the Planck likelihood? (b) What is this comparison expected to look like for future spectroscopic surveys?

\footnotetext{
${ }^{1}$ Assuming the normal hierarchy (the three states satisfy the hierarchy $\left.m_{1} \lesssim m_{2} \ll m_{3}\right)$ and that one eigenstate has a zero mass. Note that an upper bound in Eq. (1) was derived without assuming the lower bound from oscillation experiments. This point will be discussed in Secs. II and III.
}

To achieve these goals we focus on two particular wellmotivated models for which the BAO or FS information is expected to be the most relevant: $\Lambda \mathrm{CDM}$ with massive neutrinos and $\Lambda \mathrm{CDM}$ with both massive neutrinos and extra relativistic degrees of freedom (parametrized by their effective number $N_{\text {eff }}$ ). These extensions of the minimal $\Lambda \mathrm{CDM}$ model can be easily accommodated by particle physics models which feature both sterile and usual massive left-handed neutrinos (see Refs. [21,22] for reviews). The measurement of $N_{\text {eff }}$ with LSS data has been the subject of intense work; see Ref. [23] for the theoretical motivation, Ref. [24] for forecasts of current and future experiments including BOSS + Planck, and Ref. [25] for attempts to measure $N_{\text {eff }}$ in the BAO data from BOSS. Note that for other nonminimal models, e.g., dynamical dark energy, the FS power spectrum likelihood is mostly saturated with the distance information [16], and it is not expected to perform much better than the BAO-only likelihood.

It is worth noting that combined analyses of the CMB and FS galaxy power spectrum have already been performed several times $[2,9,10,26-30]$. These analyses were based on approximate phenomenological models for the nonlinear power spectrum (or the correlation function). Even though these models capture the main qualitative effects of the nonlinear clustering and redshift-space distortions, their use can lead to systematic biases in the parameter inference. These biases may be small given the error bars of the BOSS survey, but can become significant for the future high-precision LSS surveys like Euclid [31] or DESI [32]. In this paper we reanalyze the Planck and the FS BOSS legacy data using the most advanced perturbation theory model that is available to date.

Our theoretical model is an improved version of the one-loop Eulerian perturbation theory, which includes corrections that parametrize the effects of complicated short-scale physics. These corrections can be consistently taken into account within the effective field theory framework [33,34]. This model was described in detail in Refs. [16,17,35-38]. The main difference with respect to previous studies is the implementation of infrared (IR) resummation and the presence of the so-called "counterterms." IR resummation describes the nonlinear evolution of baryon acoustic oscillations, which was independently formulated within several different but equivalent frameworks [39-45]. The major novelties compared to the previous models are that (i) the nonlinear damping applies only to the oscillating ("wiggly") part of the matter power spectrum, (ii) it does not require any fitting parameters, and (iii) it includes corrections beyond the commonly used exponential suppression. As for the counterterms, their presence is required in order to capture the effects of poorly known short-scale physics $[33,34,46]$ on the long-wavelength fluctuations. In particular, these corrections provide an effective description of the baryonic 
feedback [47], higher-derivative and velocity biases [48], and the redshift-space distortions [35] including the socalled "fingers-of-God" effect [49].

This paper is structured as follows. We discuss our methodology, data sets, and the treatment of massive neutrinos in Sec. II. Section III contains our main results. In Sec. IV we present a mock analysis of the simulated BOSS data that quantifies the amount of information from the BAO and FS measurements. Finally, in Sec. V we draw our conclusions.

\section{METHODOLOGY}

In our main analysis the Markov chain Monte Carlo (MCMC) chains sample seven cosmological parameters of the minimal $\Lambda \mathrm{CDM}$ with massive neutrinos $\left(\omega_{b}, \omega_{\mathrm{CDM}}\right.$, $\left.\theta_{s}, A_{s}, \tau, n_{s}, M_{\text {tot }}\right)$, where $\omega_{b}$ and $\omega_{\mathrm{CDM}}$ are the physical densities of baryons and dark matter, respectively, $\theta_{s}$ is the angular acoustic scale of the $\mathrm{CMB}, A_{s}$ and $n_{s}$ are the amplitude and the tilt of the primordial spectrum of scalar fluctuations, $\tau$ denotes the reionization optical depth, and $M_{\text {tot }}$ is the sum of neutrino mass eigenstates. Additionally, we run an analysis with varied $N_{\text {eff }}$ which was fixed to the standard value 3.046 in the baseline run. Throughout this paper we approximate the neutrino sector with three degenerate massive states. This approximation is very accurate for both current and future surveys. The difference between the exact mass splittings and the degenerate-state approximation is negligible once the proper lower priors are imposed [12,14,50,51].

From the Planck side, we use the baseline TTTEEE + low $\ell+$ lensing likelihood from the 2018 data release [1] as implemented in MONTEPYTHON V3.0 [52]; see Ref. [53] for likelihood details. In addition to the cosmological parameters, we also vary 21 nuisance parameters that describe foregrounds, beam leakage, and other instrumental effects [53]. One difference with respect to the baseline Planck analysis is that we model the nonlinear corrections to the CMB lensing potential with one-loop perturbation theory. The reason is that the one-loop power spectrum captures the behavior of the matter power spectrum on mildly nonlinear scales much better than the commonly used fitting formulas like HALOFIT. Strictly speaking, the one-loop power spectrum cannot be applied to very nonlinear scales. However, for $\Lambda \mathrm{CDM}$ the one-loop power spectrum matches the HALOFIT formula with $20 \%$ accuracy down to $k \sim 1 \mathrm{hMpc}^{-1}$. Moreover, the one-loop expression is more reliable for nonminimal cosmological models, for which HALOFIT was simply not calibrated. We have run the Planck baseline analysis with both HALOFIT and one-loop perturbation theory and found identical results.

To quantify the constraining power of the BOSS FS likelihood, we compare our results with the joint analysis of the Planck and consensus BAO measurements based on the same BOSS data [2]. Note that this BAO likelihood is somewhat less constraining compared to the one used by the Planck Collaboration [1], which also included, e.g., data from Ly- $\alpha$ forest absorption lines [54] and quasar clustering [55]. The consensus BAO measurements of BOSS were obtained by the so-called density field reconstruction $[3,4]$, which sharpens the $\mathrm{BAO}$ feature but distorts the broadband shape, which is then marginalized over. ${ }^{2}$

The main analysis of this paper will be based on the fullshape galaxy power spectrum likelihood from the BOSS data release 12 (DR12) (year 2016) [2], which includes the monopole and quadrupole moments at wave numbers up to $k_{\max }=0.25 \mathrm{~h} / \mathrm{Mpc}$. Details of this likelihood can be found in Ref. [2]. The BOSS DR12 includes four independent data sets corresponding to different galaxy populations observed across two nonoverlapping redshift bins with $z_{\text {eff }}=0.38$ and $z_{\text {eff }}=0.61$. For each data set we use seven nuisance parameters to describe galaxy bias, baryonic feedback, "fingers-of-God," and other effects of poorly known short-scale physics, which totals to 28 additional free parameters in the joint BOSS FS likelihood. Our methodology for the BOSS full-shape analysis is identical to the one used in Ref. [16], where one can find further details of the theoretical model, covariance matrix, and the window function treatment. Additionally, in this work we account for fiber collisions by implementing the effective window method [61]. In agreement with previous works $[16,17]$, we have found that the effect of fiber collisions is largely absorbed into the nuisance parameters and has a negligible impact on the estimated cosmological parameters. In the present analysis we ignore any correlation between the BOSS and the CMB data. The crosscorrelation of BOSS galaxies with the CMB temperature has not yet been detected [62], while the correlation with the CMB lensing is small on the mildly nonlinear scales [11]. Thus, treating the BOSS and Planck data as independent is a reasonable approximation given the current error bars.

The presence of massive neutrinos requires a modification of the standard perturbative approach to galaxy clustering. Neutrino free-streaming makes the growth of matter fluctuations scale dependent, which invalidates the common perturbative schemes that are based on the factorization of time evolution in the perturbation theory kernels (the so-called Einstein-de Sitter approximation). A fully consistent description requires a proper calculation of scale-dependent Green's functions; see Refs. [63,64]. However, this description is quite laborious and has not yet been extended to galaxies in redshift space. Given the error bars of the BOSS survey, one may consider the effect of massive neutrinos perturbatively and employ some

\footnotetext{
${ }^{2}$ It is worth mentioning that a promising way to extract cosmological information from galaxy catalogs is a consistent reconstruction of the full initial density field beyond the BAO [56-60].
} 
approximations. In particular, we will use standard expressions for the one-loop integrals computed in the Einsteinde Sitter (EdS) approximation, but with the exact linear power spectrum obtained in the presence of massive neutrinos [65]. For calculations based on a two-fluid extension of standard perturbation theory, this prescription has been checked to agree with the full treatment up to a few percent difference [63]. This result was recently confirmed in effective field theory in Ref. [64]. That work showed that the leading effects of nonlinear neutrino backreaction is captured by the counterterms, which also absorb the difference between proper Green's functions and the EdS approximation on large scales. We will also employ the "cb" prescription, i.e., assume that galaxies trace only dark matter and baryons, and not the total matter density that includes the massive neutrinos. This prescription was advocated on the basis of $N$-body simulations in Refs. [66,67-70]. Furthermore, Refs. [71,72] pointed out its importance for parameter inference. Following the "cb" prescription, we evaluate the loop integrals using the standard perturbation theory redshift-space kernels with the logarithmic growth rate computed only for the baryon and dark matter components. The "cb" prescription ensures that the galaxy power spectrum matches $N$-body simulations on large scales [70], where it approaches the Kaiser prediction [73] evaluated with the linear bias and logarithmic growth factor $f$ for the baryon + dark matter fluid.

Before closing this section it is worth mentioning that Refs. [74,75] found an additional scale dependence of the galaxy bias even if it is defined with respect to $\mathrm{CDM}+$ baryons. It was argued that this effect is numerically very small for standard cosmology, but it depends linearly on the neutrino density fraction just like the other effects relevant for galaxy clustering. We leave the impact of the neutrino-induced bias on cosmological parameter measurements for future study.

\section{RESULTS}

The triangle plots with posterior densities and marginalized distributions for cosmological parameters of the $\nu \Lambda \mathrm{CDM}$ model $\left(\omega_{b}, \omega_{\mathrm{CDM}}, n_{s}, H_{0}, \Omega_{m}, \sigma_{8}, M_{\text {tot }}\right)$ are shown in Fig. 1. A similar plot obtained for a model with free $N_{\text {eff }}$ (dubbed as $\nu \Lambda \mathrm{CDM}+N_{\text {eff }}$ ) is displayed in Fig. 2. For comparison, we also show the contours obtained by analyzing the Planck data only. The results of this analysis are in good agreement with the ones reported by the Planck Collaboration [1]. ${ }^{3}$ The marginalized limits are presented in Table I.

The BOSS data notably improve the limits on the latetime parameters $H_{0}, \Omega_{m}, \sigma_{8}$, and the neutrino mass $M_{\text {tot }}$. This happens mainly due to the breaking of degeneracies between $H_{0}$ and other cosmological parameters in the CMB

\footnotetext{
${ }^{3}$ https://wiki.cosmos.esa.int/planck-legacy-archive/images/b/be/ Baseline_params_table_2018_68pc.pdf.
}

likelihood. This is not surprising, as the precision of the $H_{0}$ measurement from the BOSS FS data alone rivals that of the CMB. The main improvement on the sum of neutrino masses brought by the BOSS data also comes from a better $H_{0}$ determination (this result was foreseen long ago in Ref. [76]). $H_{0}$ and $M_{\text {tot }}$ are anticorrelated in the CMB data, and the BOSS likelihood pulls $H_{0}$ to slightly higher values [16], which pushes the neutrino masses closer to the origin. However, the BOSS data at the same time prefer a somewhat low value of $\sigma_{8}$ [16] which pulls the neutrino masses in the opposite direction. This is reflected in our 95\% C.L. limit $0.16 \mathrm{eV}$, which is higher than the Planck $+\mathrm{BAO}$ measurement $0.12 \mathrm{eV}$. We stress that this relaxation does not imply that the FS data has less statistical power than the BAO. On the contrary, it is a result of taking into account new information that the BOSS clustering amplitude is lower than the Planck $+\mathrm{BAO}$ prediction.

It is important to recall that the Planck $+\mathrm{BAO}$ upper limit on the neutrino mass is driven by the so-called "lensing anomaly," which prefers an enhanced amplitude of the late-time clustering [1]. ${ }^{4}$ One way to reduce this tension is to include the information from other data sets, i.e., the clustering amplitude measurement of BOSS used in our work. However, the BAO only contains the distance information and does not directly relax the "lensing anomaly." Thus, the Planck + BAO limit should not be overinterpreted. In contrast, our new constraint, which also includes the amplitude of the power spectrum weakens the lensing anomaly and leads to a less aggressive bound.

It is instructive to see how much the neutrino mass bounds depend on the priors. Following the Planck methodology, we have imposed an unphysical zero lower limit in our baseline analysis. The rationale behind this approach is to obtain constraints directly from cosmological data regardless of particle physics priors. However, the physical priors corresponding to the normal or inverted hierarchies ( $\mathrm{NH}$ and $\mathrm{IH}$ in what follows, respectively) can notably change the result. To estimate this effect we have resampled our chains with the physical lower priors $0.06 \mathrm{eV}$ for $\mathrm{NH}$ and $0.1 \mathrm{eV}$ for $\mathrm{IH}$, which follow from the oscillation experiments [7]. We obtained the following bounds (at $95 \%$ C.L.):

$$
\begin{array}{ll}
M_{\mathrm{tot}}<0.18 \mathrm{eV} & (\mathrm{NH}, \text { Planck }+\mathrm{FS}), \\
M_{\mathrm{tot}}<0.21 \mathrm{eV} & (\mathrm{IH}, \text { Planck }+\mathrm{FS}) .
\end{array}
$$

These values can be compared with the Planck + BAO results which were extracted from our chains by a similar resampling,

\footnotetext{
${ }^{4}$ One way to see this is to compare the error on the actual $M_{\text {tot }}$ measurement of Planck with the one obtained from the simulated data, e.g., in Ref. [38]. The error from the simulated data is typically twice as large, which suggests that the Planck measurement is driven by a tension in the data.
} 


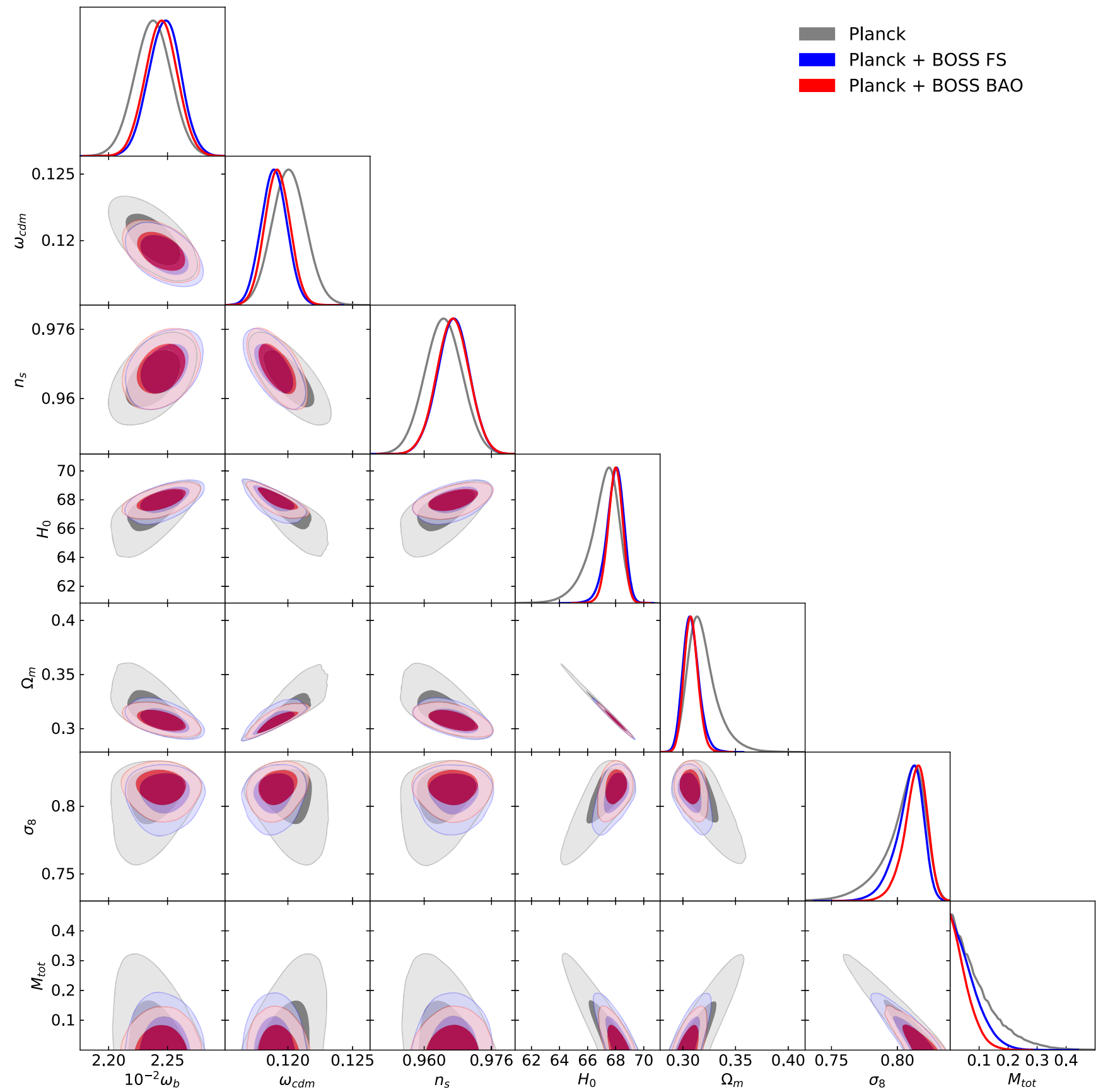

FIG. 1. Marginalized one-dimensional posterior distribution and two-dimensional probability contours (at the $68 \%$ and $95 \%$ C.L.) for the parameters of the $\Lambda \mathrm{CDM}$ model with varied neutrino masses. $N_{\text {eff }}$ is fixed to the standard model value $3.046 . H_{0}$ is quoted in $\mathrm{km} / \mathrm{s} /$ $\mathrm{Mpc}$, while $M_{\text {tot }}$ is quoted in $\mathrm{eV}$.

$$
\begin{array}{ll}
M_{\mathrm{tot}}<0.15 \mathrm{eV} & (\mathrm{NH}, \text { Planck }+\mathrm{BAO}), \\
M_{\mathrm{tot}}<0.18 \mathrm{eV} & (\mathrm{IH}, \text { Planck }+\mathrm{BAO}) .
\end{array}
$$

As far as the base cosmological parameters are concerned, the improvement from the FS data (which embody the unreconstructed $\mathrm{BAO}$ ) for $\nu \Lambda \mathrm{CDM}$ is comparable to that from the reconstructed $\mathrm{BAO}$ measurements [1]. This reflects the fact that the shape of the matter power spectrum does not contribute significantly to the cosmological constraints on the physical densities of baryons and dark matter, which are dominated by Planck.

One may expect that the shape information can be more important in the model with additional relativistic degrees of freedom. However, in this model the CMB degeneracy direction in the $\omega_{\mathrm{CDM}}-H_{0}$ plane changes its orientation compared to the base $\Lambda \mathrm{CDM}$ and accidentally becomes aligned with the degeneracy direction of the 


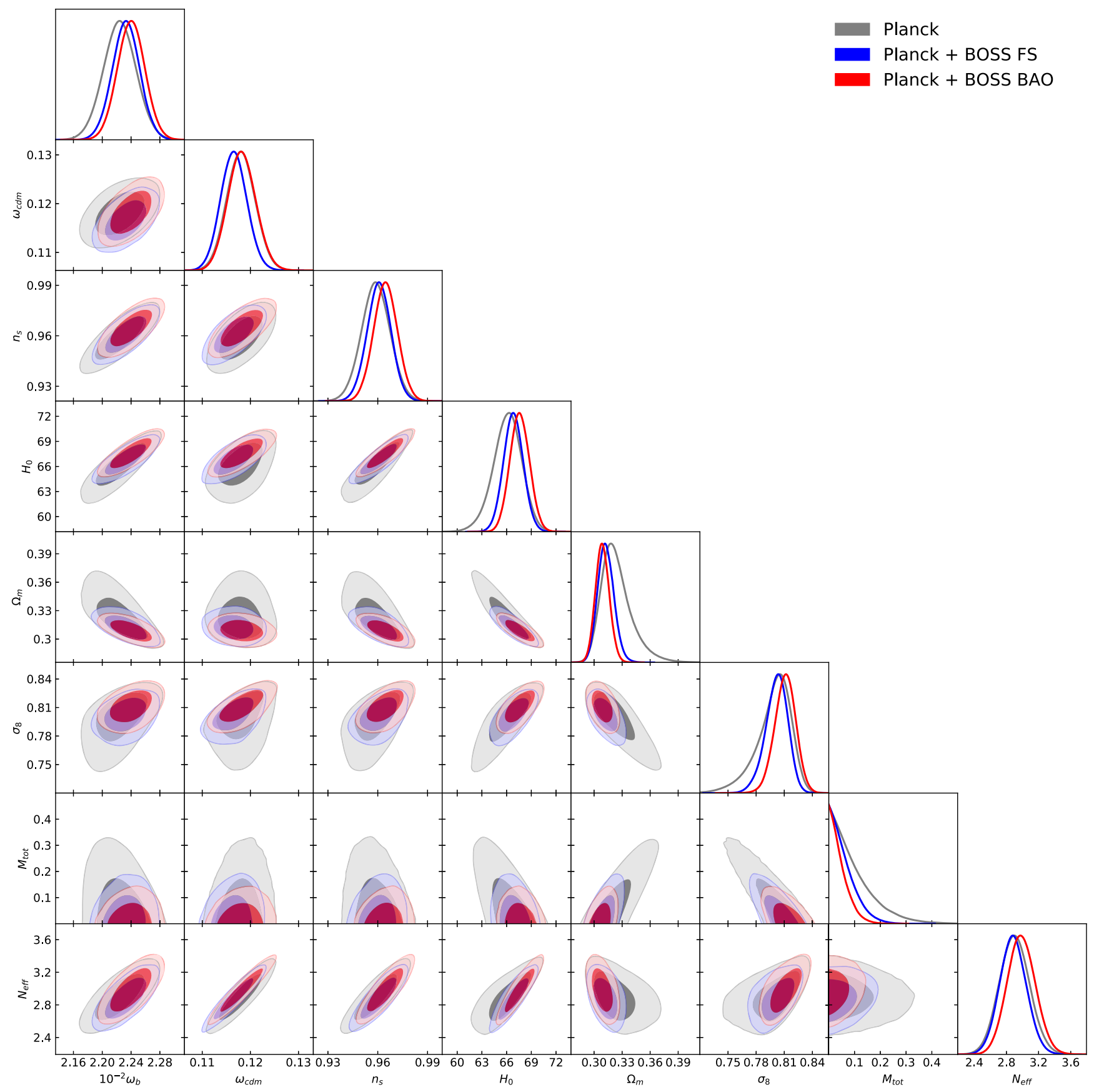

FIG. 2. Marginalized one-dimensional posterior distribution and two-dimensional probability contours (at the $68 \%$ and $95 \%$ C.L.) for the parameters of the $\Lambda \mathrm{CDM}$ model with both varied neutrino masses and the effective number of relativistic degrees of freedom $N_{\text {eff }}$. $H_{0}$ is quoted in $\mathrm{km} / \mathrm{s} / \mathrm{Mpc}$, while $M_{\text {tot }}$ is quoted in $\mathrm{eV}$.

BOSS data. Due to this coincidence the parameter degeneracies from the two data sets are not broken, and the improvement from their combination is quite modest. Importantly, the posterior contour in the $\omega_{\mathrm{CDM}}-H_{0}$ plane is shifted down as a consequence of the preference of the BOSS data for low $\omega_{\mathrm{CDM}}$ [16]. This also produces some $\sim 0.5 \sigma$ shifts in cosmological parameters as compared to the Planck + BAO analysis. In particular, we find

$$
\left.\begin{array}{rl}
N_{\text {eff }} & =2.88 \pm 0.17 \\
H_{0} & =66.8 \pm 1.2
\end{array}\right\} \quad(68 \%, \text { Planck }+\mathrm{FS}),
$$

which can be contrasted with

$$
\left.\begin{array}{c}
N_{\text {eff }}=2.99 \pm 0.17 \\
H_{0}=67.6 \pm 1.2
\end{array}\right\} \quad(68 \%, \text { Planck }+ \text { BAO }),
$$


TABLE I. Mean values and $68 \%$ C.L. minimum credible intervals for the parameters of the $\nu \Lambda$ CDM (left three columns) and $\nu \Lambda \mathrm{CDM}+N_{\text {eff }}$ (right three columns) models as extracted from the Planck, Planck + BAO, and Planck + FS data, presented as "mean ${ }_{-1 \sigma}^{+1 \sigma}$." For $M_{\text {tot }}$ we quote the $95 \%$ C.L. upper limit in units of eV. $H_{0}$ is quoted in $\mathrm{km} / \mathrm{s} / \mathrm{Mpc}$.

\begin{tabular}{|c|c|c|c|c|c|c|}
\hline \multirow[b]{2}{*}{ Parameter } & \multicolumn{3}{|c|}{$\nu \Lambda \mathrm{CDM}$} & \multicolumn{3}{|c|}{$\nu \Lambda \mathrm{CDM}+N_{\mathrm{eff}}$} \\
\hline & Planck & Planck + BAO & Planck + FS & Planck & Planck + BAO & Planck + FS \\
\hline $100 \omega_{b}$ & $2.238_{-0.015}^{+0.016}$ & $2.245_{-0.014}^{+0.014}$ & $2.247_{-0.013}^{+0.015}$ & $2.224_{-0.023}^{+0.023}$ & $2.240_{-0.019}^{+0.019}$ & $2.233_{-0.019}^{+0.019}$ \\
\hline$\omega_{\mathrm{CDM}}$ & $0.1201_{-0.0014}^{+0.0013}$ & $0.11919_{-0.00099}^{+0.00099}$ & $0.11893_{-0.001}^{+0.00097}$ & $0.1181_{-0.0031}^{+0.003}$ & $0.1182_{-0.0031}^{+0.0029}$ & $0.1166_{-0.0028}^{+0.0026}$ \\
\hline $100 \theta_{s}$ & $1.04187_{-0.00030}^{+0.00030}$ & $1.04195_{-0.00029}^{+0.00029}$ & $1.04196_{-0.00028}^{+0.00028}$ & $1.04220_{-0.00054}^{+0.00051}$ & $1.04210_{-0.00052}^{+0.0005}$ & $1.04234_{-0.0005}^{+0.00049}$ \\
\hline$\tau$ & $0.0543_{-0.0079}^{+0.0074}$ & $0.05556_{-0.0076}^{+0.007}$ & $0.05539_{-0.0072}^{+0.0074}$ & $0.05341_{-0.008}^{+0.0074}$ & $0.05516_{-0.0078}^{+0.0072}$ & $0.05409_{-0.0075}^{+0.0073}$ \\
\hline $\ln \left(10^{10} A_{s}\right)$ & $3.045_{-0.016}^{+0.014}$ & $3.045_{-0.015}^{+0.014}$ & $3.044_{-0.014}^{+0.014}$ & $3.037_{-0.018}^{+0.018}$ & $3.042_{-0.017}^{+0.017}$ & $3.035_{-0.017}^{+0.016}$ \\
\hline$n_{s}$ & $0.9646_{-0.0045}^{+0.0045}$ & $0.9669_{-0.0039}^{+0.0039}$ & $0.967_{-0.004}^{+0.0038}$ & $0.9588_{-0.0087}^{+0.0087}$ & $0.9647_{-0.0074}^{+0.0073}$ & $0.9608_{-0.0072}^{+0.0074}$ \\
\hline$M_{\text {tot }}$ & $<0.26$ & $<0.12$ & $<0.16$ & $<0.27$ & $<0.12$ & $<0.16$ \\
\hline$N_{\text {eff }}$ & & fixed 3.046 & & $2.90_{-0.19}^{+0.19}$ & $2.99_{-0.17}^{+0.17}$ & $2.88_{-0.17}^{+0.17}$ \\
\hline$\Omega_{m}$ & $0.3188_{-0.016}^{+0.0091}$ & $0.3078_{-0.0071}^{+0.0060}$ & $0.3079_{-0.0085}^{+0.0065}$ & $0.324_{-0.019}^{+0.011}$ & $0.3090_{-0.0076}^{+0.007}$ & $0.3127_{-0.0091}^{+0.0080}$ \\
\hline$H_{0}$ & $67.14_{-0.72}^{+1.3}$ & $67.97_{-0.49}^{+0.56}$ & $67.95_{-0.52}^{+0.66}$ & $66.1_{-1.6}^{+1.9}$ & $67.6_{-1.2}^{+1.2}$ & $66.8_{-1.2}^{+1.2}$ \\
\hline$\sigma_{8}$ & $0.8053_{-0.0091}^{+0.019}$ & $0.8135_{-0.0073}^{+0.01}$ & $0.8087_{-0.0072}^{+0.012}$ & $0.798_{-0.013}^{+0.022}$ & $0.811_{-0.011}^{+0.012}$ & $0.8015_{-0.011}^{+0.013}$ \\
\hline
\end{tabular}

where $H_{0}$ is quoted in $\mathrm{km} / \mathrm{s} / \mathrm{Mpc}$. Note that the FS and BAO data pull the mean of $N_{\text {eff }}$ in different directions. Moreover, unlike the FS data, the BAO data notably shift the means of other parameters, e.g., $\omega_{\mathrm{CDM}}$ and $H_{0}$. This shows that the full-shape and BAO data (i) contain different information, and (ii) have similar statistical powers in combination with Planck. The interpretation of these results is that most of the improvement in the joint constraint comes from the breaking of geometric degeneracy between $H_{0}$ and other cosmological parameters. Both the BAO and FS data have the same amount of geometric information that primarily constrains $H_{0}$ for the models that we considered [16], and hence the error bars are very similar. However, the additional shape and clustering amplitude information contained in the FS data are not negligible, and their addition leads to $\sim 0.5 \sigma$ shifts of the Planck + FS posteriors compared to Planck + BAO.

Finally, let us remark that we varied $N_{\text {eff }}$ together with the neutrino mass, but this choice does not degrade our limits compared to a fit with fixed $M_{\text {tot }}$. The reason is that the Planck data itself clearly distinguish between the two effects because the error bars from the joint $M_{\text {tot }}+N_{\text {eff }}$ fit are the same as in the individual $M_{\text {tot }}$ and $N_{\text {eff }}$ runs [1]. This is also true for the BOSS likelihood, as we have obtained identical constraints on $M_{\text {tot }}$ that are twice as strong as Planck both with fixed and varied $N_{\text {eff }}$. This suggests that the two effects are clearly discriminated by the BOSS data too.

\section{WIGGLES VS BROADBAND}

In the previous section we presented results for the two different analyses: Planck + BAO and Planck + FS. As argued in the Introduction, the information extracted from the galaxy clustering in these two methods is quite different, yet the error bars in the two analyses are identical. ${ }^{5}$ This is a very striking feature of our results and it requires an explanation. In this section we investigate the information content in the BAO and FS analyses in detail and show that the identical error bars are just a coincidence for the given volume of the BOSS survey and the given BAO reconstruction efficiency. We will argue that for larger future spectroscopic surveys the FS analysis will eventually be more powerful in constraining the cosmological parameters.

In order to compare the amount of information in the reconstructed $\mathrm{BAO}$ wiggles with the amount of information in the full-shape power spectrum (which embeds the unreconstructed $\mathrm{BAO}$ ), we analyzed several sets of the simulated mock data which mimic the actual BOSS sample, but have different amplitudes of the BAO wiggles. This exercise is analogous to that performed in Ref. [16], where one can find further details of our mock data set. We generated four sets of power spectra multipoles for the low$z\left(z_{\mathrm{eff}}=0.38\right)$ DR12 North Galactic Cap (NGC) sample with a different amount of BAO damping and analyzed them using the same pipeline, appropriately modified in each case. The mock data were assigned the covariance of the real sample. For clarity, we analyze the mock BOSS data per se, i.e., without the Planck likelihood. ${ }^{6}$

\footnotetext{
${ }^{5}$ As discussed in the previous section, the $95 \%$ upper bound on $M_{\text {tot }}$ in the FS analysis is larger. However, this is due to the low $\sigma_{8}$ measured by BOSS compared to the CMB, which pulls the upper bound to the larger value. This does not happen in the BAO analysis, since $\sigma_{8}$ is not measured.

${ }^{6}$ There are two effects that contribute to the constraints in combination with Planck: the size of the error bar itself, and the orientation of the posterior contours (e.g., $H_{0}-\omega_{\mathrm{CDM}}$ ) with respect to the Planck ones, which is different for BAO and FS.
} 
To understand our method, recall that the BAO damping at leading order can be described as

$$
P_{\mathrm{IR} \mathrm{res}, \mathrm{LO}}(k, \mu)=P_{\mathrm{nw}}+\mathrm{e}^{-\Sigma^{2} k^{2}} P_{\mathrm{w}}
$$

where $P_{\mathrm{nw}}$ and $P_{\mathrm{w}}$ are the de-wiggled broadband and wiggly parts of the linear power spectrum, respectively. We also introduced $\mu \equiv \cos (\mathbf{z}, \mathbf{k})$, where $\mathbf{z}$ is the line-of-sight vector. The theoretical prediction for the damping factor $\Sigma$ is given by $[42,44,45]$,

$$
\begin{aligned}
\Sigma^{2}= & \Sigma_{\mathrm{NL}}^{2} \equiv\left(1+f \mu^{2}(2+f)\right) \\
& \times \frac{4 \pi}{3} \int_{0}^{k_{s}} d q P_{\mathrm{nw}}(q)\left[1-j_{0}\left(q r_{d}\right)+2 j_{2}\left(q r_{d}\right)\right],
\end{aligned}
$$

where $f$ is the logarithmic growth factor, $j_{\ell}(x)$ are spherical Bessel functions, $k_{S}$ is an arbitrary scale separating the resummed soft modes, and $r_{d}$ is the sound horizon at the drag epoch. We emphasize that the leading-order expression (6) has a non-negligible dependence on $k_{S}$, which greatly decreases after computing the one-loop correction to Eq. (6) [44]. Note that we use Eq. (6) only for illustration purposes. In the actual analysis we compute the full oneloop IR-resummed expression with appropriately modified values of $\Sigma$.

The four mock samples are characterized by four different BAO damping factors $\Sigma=\infty, \Sigma_{\mathrm{NL}}, \Sigma_{\mathrm{NL}} / 2,0$, where $\Sigma_{\mathrm{NL}}$ is the theoretically predicted amount of BAO damping (7). The first case corresponds to the pure broadband information without any wiggles. The second case mimics the real physical situation and reproduces the actual constraints from the FS analysis of the BOSS low- $z$ NGC data sample. The third situation corresponds to the combination of the broadband with the standard BAO reconstruction, which reduces the damping by a factor of 2 [77]. Finally, the fourth scenario features the full BAO wiggles, which are not affected by the nonlinear smearing. This case corresponds to the joint analysis of the broadband+optimally reconstructed BAO wiggles, which is the best case scenario for $\mathrm{BAO}$ reconstruction. ${ }^{\text {? }}$

For the purposes of this section, we focus on the set of cosmological parameters $\left(H_{0}, \omega_{\mathrm{CDM}}, \sigma_{8}\right)$ and use the Planck priors for $\omega_{b}$ and $n_{s}$. We fixed $M_{\text {tot }}=0$ in our simulated

\footnotetext{
${ }^{7}$ The standard reconstruction technique does not fully restore the linear amplitude of the BAO wiggles [77]. However, more sophisticated methods have the potential to achieve almost optimal efficiency. One example is the iterative reconstruction, so far applied only to dark matter in real space [6]. Another example is the neural-network-based algorithm of Ref. [57], which is close to optimal for halos. It will be interesting to see how much these more advanced approaches can improve BAO reconstruction in the realistic case of biased tracers in redshift space.
}

data. The chosen fitting parameters represent three different sources of information encoded in the power-spectrum multipoles: the geometric distance $\left(H_{0}\right)$, shape of the transfer functions $\left(\omega_{\mathrm{CDM}}\right)$, and redshift-space distortions $\left(\sigma_{8}\right) .{ }^{8}$ The results of our analysis are displayed in Fig. 3 and Table II. We also show the derived parameter $\Omega_{m}$, which comes from a combination of the shape and distance information.

The relative importance of the $\mathrm{BAO}$ wiggles compared to the broadband can be assessed by comparing the results for the four different mock data sets. The first observation is that the BAO wiggles significantly affect only the $H_{0}$ measurement. There is no improvement in $\omega_{\mathrm{CDM}}$ between the reconstructed and unreconstructed cases, and a very slight error bar reduction for the clustering amplitude $\sigma_{8}$. The second observation is that the $H_{0}$ constraint improves by $\sim 40 \%$ (i.e., the error reduces by $\sim \sqrt{2}$ ) for $\Sigma=\Sigma_{\mathrm{NL}} / 2$ compared to the $\Sigma=\Sigma_{\mathrm{NL}}$ case. Thus, we can conclude that the reconstructed high- $k \mathrm{BAO}$ wiggles measure $H_{0}$ with a similar precision as the FS data. This is precisely related to our result that the $\mathrm{BAO}$ and FS data have a similar amount of geometric information. However, this is just a coincidence. Even small modifications in the setup can drastically change the conclusions. For example, in the case of the ideal BAO reconstruction the error on $H_{0}$ is smaller by more than a factor of 2 compared to the standard FS analysis. This result suggests that $H_{0}$ error bars are very sensitive to the efficiency of BAO reconstruction. While the $\Sigma=0$ limit is probably impossible to get in practice, any improvement in the reconstruction algorithm can potentially be very important for the BOSS data analysis.

Another relevant parameter in this discussion is the volume of the survey. Smaller statistical errors can significantly improve the cosmological constraints thanks to the degeneracy breaking among many nuisance parameters needed to describe the broadband. ${ }^{9}$ Furthermore, larger surveys include higher redshifts, where the BAO peak is much less damped. Thus, the expectation is that for large enough volumes the FS should eventually win over the BAO-only analysis.

This expectation was confirmed in the analysis of Ref. [38]. The authors of that work carried out an analysis of the joint FS + BAO mock data of a Euclid-like survey (whose volume is roughly 10 times larger than BOSS) which is identical to the analysis of this section. It has been shown that even in the ideal case of the $100 \%$ efficient BAO reconstruction the error bar on $H_{0}$ improves only by $\lesssim 30 \%$ compared to the FS constraints, which should be contrasted

\footnotetext{
${ }^{8}$ In the $\Lambda \mathrm{CDM}$ model the logarithmic growth rate $f$ is fixed by $\Omega_{m}$ and $H_{0}$ (modulo a small effect due to massive neutrinos), which are extracted from the monopole.

${ }^{9}$ In such cases the improvement can be much better than naive estimates using the mode counting.
} 


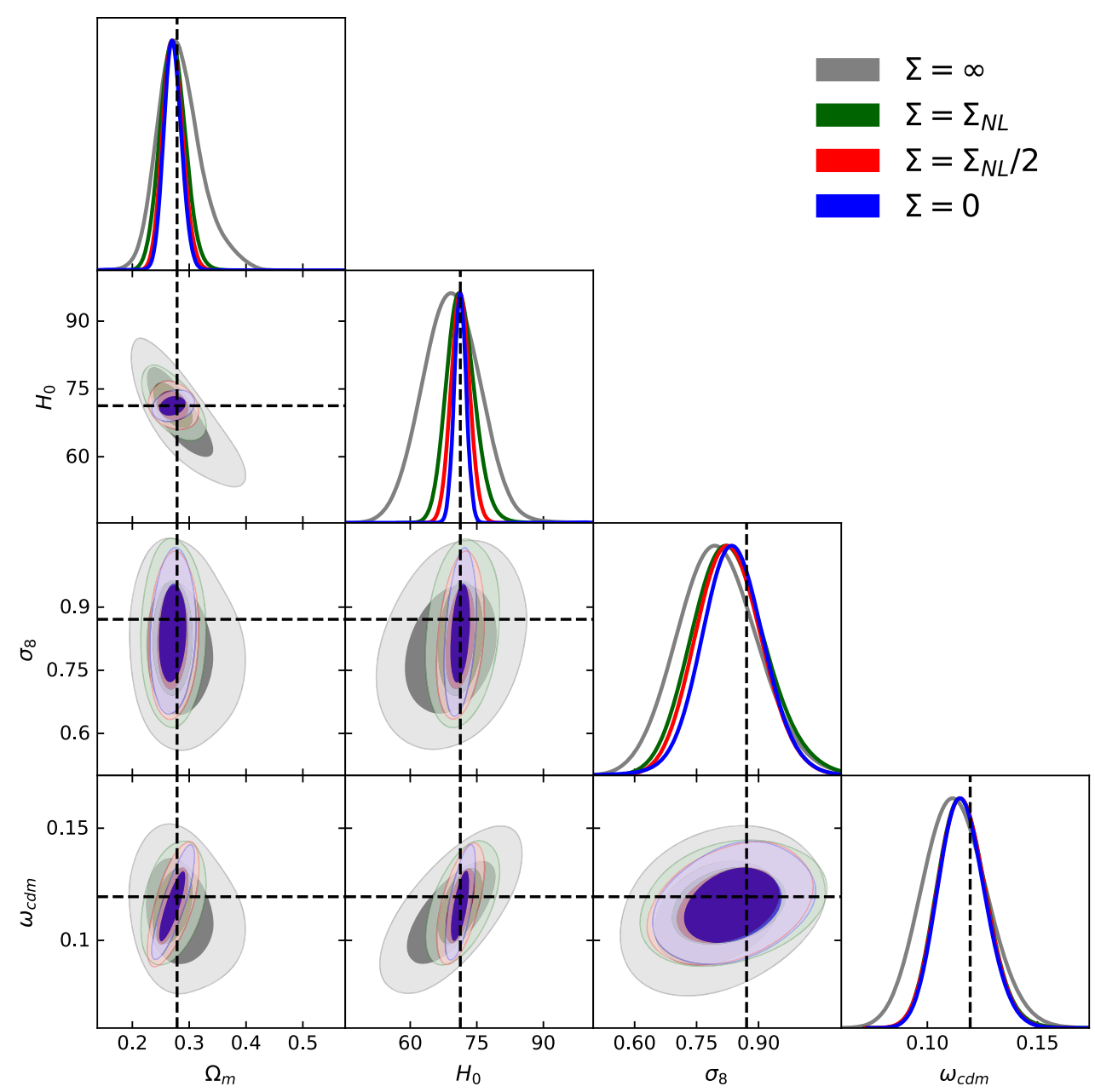

FIG. 3. Marginalized one-dimensional posterior distribution and two-dimensional probability contours (at the $68 \%$ and $95 \%$ C.L.) for the parameters of simulated mock BOSS data. The four cases correspond to different amounts of BAO smoothing; see the main text for further details.

with the $\sim 100 \%$ improvement for the BOSS volume. Repeating the analysis of Ref. [38] for a more realistic case of a $50 \%$ efficient reconstruction, we found the improvement for the Euclid data to be marginal $(\lesssim 10 \%)$, which can be contrasted with the $\sim 40 \%$ gain for the BOSS

TABLE II. Mean values and $68 \%$ C.L. minimum credible intervals for the parameters extracted from the simulated data mocking the BOSS DR12 low- $z$ NGC sample. The values of $H_{0}$ are quoted in units of $\mathrm{km} / \mathrm{s} / \mathrm{Mpc}$.

\begin{tabular}{lcccc}
\hline \hline Param. & $\Sigma=\infty$ & $\Sigma=\Sigma_{\mathrm{NL}}$ & $\Sigma=\Sigma_{\mathrm{NL}} / 2$ & $\Sigma=0$ \\
\hline$\omega_{\mathrm{CDM}}$ & $0.112_{-0.015}^{+0.014}$ & $0.116_{-0.011}^{+0.010}$ & $0.116_{-0.011}^{+0.010}$ & $0.116_{-0.011}^{+0.010}$ \\
$H_{0}$ & $69.3_{-6.1}^{+6.3}$ & $71.4_{-3.4}^{+2.9}$ & $71.3_{-2.1}^{+1.9}$ & $71.3_{-1.4}^{+1.2}$ \\
$\sigma_{8}$ & $0.802_{-0.100}^{+0.091}$ & $0.828_{-0.092}^{+0.082}$ & $0.828_{-0.075}^{+0.076}$ & $0.837_{-0.071}^{+0.072}$ \\
$\Omega_{m}$ & $0.284_{-0.074}^{+0.031}$ & $0.271_{-0.021}^{+0.021}$ & $0.271_{-0.018}^{+0.017}$ & $0.271_{-0.016}^{+0.015}$ \\
\hline \hline
\end{tabular}

volume. ${ }^{10}$ These results are not very surprising, and similar trends have already been seen in several other forecasts (see, for instance, the $\mathrm{BAO}$ and broadband comparison in Ref. [32]). To clearly illustrate that the cosmological constraints from Planck + FS for a Euclid-like survey will be much better than the constraints from Planck + Euclid BAO alone, we have done an MCMC forecast based on the same pipeline used to analyze the actual BOSS data. The details of this forecast are presented in the Appendix.

\footnotetext{
${ }^{10}$ The inclusion of the higher-order $n$-point functions further strengthens the case for the full-shape analysis. For instance, Ref. [38] showed that the combination of the one-loop power spectrum and tree-level bispectrum monopole can lead to better constraints than the best case power spectrum analysis with the optimally reconstructed BAO wiggles. One may expect an even greater benefit from the addition of the higher-multipole moments of the bispectrum [78].
} 
In conclusion, by comparing the amount of information in the BAO and FS analyses in detail, we find that the similarity between the two in combination with Planck is just a coincidence of the BOSS survey volume and efficiency of the current reconstruction algorithms. Our analysis suggests two main conclusions: (a) better reconstruction algorithms or an optimal combination of the FS and BAO analyses can lead to tighter constraints on cosmological parameters using the same BOSS data, and (b) the full-shape power spectrum data will supersede the $\mathrm{BAO}$ measurements in the era of future galaxy surveys.

\section{CONCLUSIONS}

We have presented a joint analysis of the final Planck $\mathrm{CMB}$ and BOSS galaxy power spectrum data. Our main results include new limits on the parameters of the minimal $\Lambda \mathrm{CDM}$ model, neutrino masses, and the number of effective relativistic degrees of freedom. The key new feature of our work is the use of a new BOSS full-shape power spectrum likelihood, which is based on an improved perturbation theory model. This model consistently accounts for nonlinearities of the underlying dark matter fluid, galaxy bias, redshift-space distortions, and nonlinear effects of large-scale bulk flows.

We showed that the addition of the BOSS FS data improves the Planck-only constraints. The results for the minimal $\Lambda \mathrm{CDM}$ model with varied $M_{\text {tot }}$ are very similar to the standard Planck + BAO analysis. For the model with additional relativistic degrees of freedom the FS and BAO data yield comparable statistical improvements but shift the posterior in different directions. We argued that this is the effect of the additional full-shape information beyond the geometric location of the BAO.

When combined with Planck, the cosmological information in the shape of the BOSS galaxy power spectrum turned out to be comparable to the pure geometric information extracted form the reconstructed $\mathrm{BAO}$ peak for the cosmological models considered in this paper. However, the FS measurement will become more powerful than BAO in the era of future galaxy surveys, even for constraining vanilla cosmological scenarios. Importantly, the precision of the shape parameter measurements from these surveys will be comparable to that of Planck, and the combination of the two will reduce the error bars by a factor of a few due to degeneracy breaking [38]. This effect will be essential for the future neutrino mass measurements [32,38,79-83]. The presented constraints set a reference mark for future LSS and CMB observations that will surpass Planck and BOSS.

\section{ACKNOWLEDGMENTS}

We are indebted to Anton Chudaykin for his collaboration on the initial stages of this projects. We are grateful to Uroš Seljak for his comments on the draft of this paper. We thank Thejs Brinckmann, Emanuele Castorina, Antony Lewis, and Inar Timiryasov for valuable discussions. All numerical analyses of this work were performed on the Helios cluster at the Institute for Advanced Study. M. I. is partially supported by the Simons Foundation's Origins of the Universe program and by the RFBR Grant No. 20-0200982 A. M. Z. is supported by NSF Grants No. AST1409709, PHY-1521097, and PHY-1820775, the Canadian Institute for Advanced Research (CIFAR) Program on Gravity and the Extreme Universe, and the Simons Foundation Modern Inflationary Cosmology initiative. Parameter estimates presented in this paper were obtained with a modified version of the CLASS code [84] interfaced with the MONTEPYTHON MCMC sampler [52,85]. Perturbation theory integrals were evaluated using the method described in Ref. [86]. The plots with posterior densities and marginalized limits were generated with the latest version of the getdist package, ${ }^{11}$ which is part of the CosmoMC code $[87,88]$.

\section{APPENDIX: FULL-SHAPE VS BAO IN COMBINATION WITH PLANCK FOR A EUCLID-LIKE SURVEY}

The goal of this appendix is to show that for future surveys the power spectrum shape will be more important than just the BAO likelihood (both in combination with Planck).

Our methodology is identical to that in Ref. [38], where one can find all of the details of our analysis. We simulate the mock data on the redshift-space galaxy power spectrum from the future Euclid spectroscopic survey. From the Planck side, we use the realistic mock likelihood introduced in Ref. [51] and implemented in MONTEPYTHON V3.0 [52]. Our fiducial cosmological model is the base $\Lambda \mathrm{CDM}$ model with the best-fit Planck 2018 cosmological parameters, supplemented with one massive neutrino of $100 \mathrm{meV}$. This model is used across all simulated likelihoods. Note that the Planck mock likelihood yields somewhat weaker constraints on $H_{0}$ and $M_{\text {tot }}$ due to the absence of the discrepancy between the high- $\ell$ and low- $\ell$ likelihoods present in the real data [1] (sometimes referred to as the "lensing tension").

As far as the mock BAO likelihood is concerned, we use a $0.1 \%$ Gaussian prior on the isotropic BAO parameter $r_{d} / D_{V}$ centered at the value computed in the fiducial cosmology at $z_{\text {eff }}=0.61$ (the lowest redshift bin of the survey). Here we introduce the sound horizon at decoupling $r_{d}$ and the volume-averaged distance,

\footnotetext{
${ }^{11}$ https://getdist.readthedocs.io/en/latest/.
} 
$D_{V}(z)=\left(z D_{M}^{2}(z) / H(z)\right)^{1 / 3}$,

where $D_{M} \equiv \int_{0}^{z} \frac{d z^{\prime}}{H\left(z^{\prime}\right)}$,

and $H^{2}(z)=H_{0}^{2}\left(\Omega_{m}(1+z)^{3}+1-\Omega_{m}\right)$.

The anisotropic BAO parameter $D_{M}(z) H(z)$ does not contain any additional information in the $\Lambda$ CDM model [16].
We take the variance on $r_{d} / D_{V}$ from the mock Euclid BAO forecast of Ref. [80], which optimistically predicted the cumulative error on the isotropic BAO measurement to be $0.15 \%$. Since the dependence of the volume-averaged distance on $\Omega_{m}$ is extremely weak [16], the use of $r_{d} / D_{V}$ at one redshift bin with a cumulative error from the whole survey provides a very good approximation to the full BAO measurements across all redshifts.

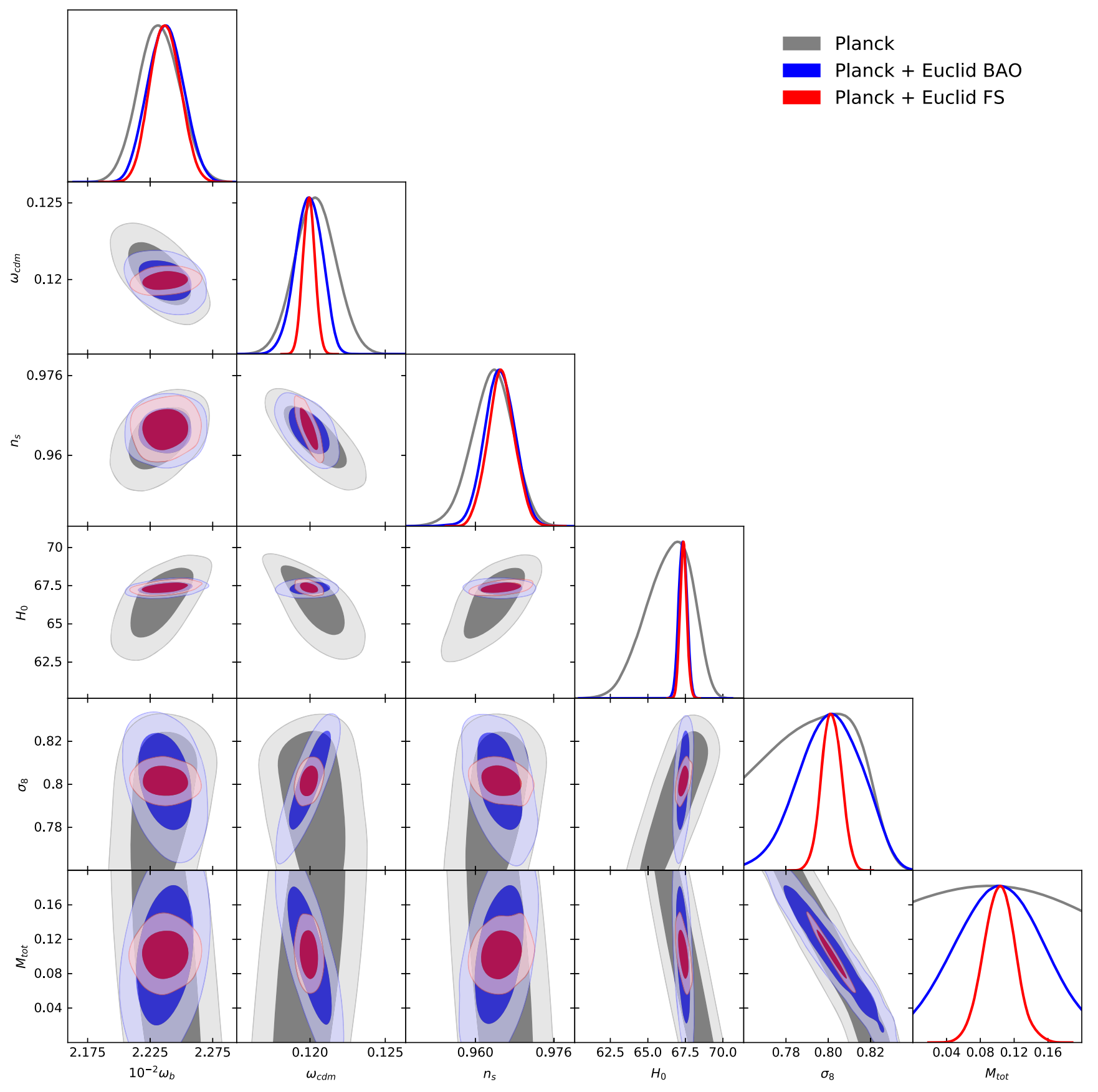

FIG. 4. Marginalized one-dimensional posterior distribution and two-dimensional probability contours (at the 68\% and 95\% C.L.) for cosmological parameters from the joint analysis of the simulated mock Planck and Euclid data. 
To understand the impact of the shape information, we combine the BAO measurements with our Planck mock data and compare it with the results from the joint Planck + FS power spectrum likelihood for a Euclid-like survey. The means and $1 \sigma$ errors on relevant cosmological parameters are displayed in Table III and the corresponding two-dimensional marginalized distribution is shown in Fig. 4. We clearly see that the addition of the FS data allows one to break degeneracies of the $\mathrm{CMB}$ data and significantly improve the constraints on all cosmological parameters. In particular, one observes a strong improvement on $H_{0}, \omega_{\mathrm{CDM}}, n_{s}, \sigma_{8}$, and $M_{\text {tot }}$. However, if we only use the geometric information from the BAO likelihood, the constraints are notably worse, especially on $M_{\mathrm{tot}}, \omega_{\mathrm{CDM}}$, and $\sigma_{8}$. We clearly see that the clustering amplitude information extracted in the FS analysis is crucial for the neutrino mass measurement.
TABLE III. Mean values and $68 \%$ C.L. minimum credible intervals for the parameters extracted from the simulated Euclid mock BAO and full-shape data combined with the mock Planck likelihood. The results for $H_{0}$ are quoted in units of $\mathrm{km} / \mathrm{s} / \mathrm{Mpc}$, while those for $M_{\text {tot }}$ are in $\mathrm{meV}$.

\begin{tabular}{lccc}
\hline \hline Params. & Planck & Planck + BAO & Planck + FS \\
\hline$H_{0}$ & $67.37_{-1.2}^{+1.8}$ & $67.33_{-0.27}^{+0.27}$ & $67.35_{-0.22}^{+0.22}$ \\
$\ln \left(10^{10} A_{s}\right)$ & $3.045_{-0.0087}^{+0.0087}$ & $3.045_{-0.0087}^{+0.0087}$ & $3.044_{-0.0082}^{+0.0082}$ \\
$\omega_{\mathrm{CDM}}$ & $0.12_{-0.0013}^{+0.0013}$ & $0.12_{-8.8 \times 10^{-4}}^{+8.8 \times 10^{-4}}$ & $0.12_{-4 \times 10^{-4}}^{+4 \times 10^{-4}}$ \\
$100 \omega_{b}$ & $2.232_{-0.016}^{+0.016}$ & $2.237_{-0.014}^{+0.014}$ & $2.237_{-0.012}^{+0.012}$ \\
$\tau_{\text {reio }}$ & $0.058_{-0.0045}^{+0.0045}$ & $0.055_{-0.0044}^{+0.0044}$ & $0.055_{-0.0044}^{+0.0044}$ \\
$n_{s}$ & $0.9634_{-0.0042}^{+0.0042}$ & $0.9649_{-0.0031}^{+0.0031}$ & $0.9653_{-0.0027}^{+0.0027}$ \\
$M_{\text {tot }}$ & $<140(68 \%$ C.L. $)$ & $107 \pm 52$ & $103 \pm 19$ \\
$\sigma_{8}$ & $0.7824_{-0.018}^{+0.037}$ & $0.8_{-0.014}^{+0.014}$ & $0.8_{-0.0046}^{+0.0046}$ \\
\hline \hline
\end{tabular}

[1] N. Aghanim et al. (Planck Collaboration), arXiv:1807 .06209 .

[2] S. Alam et al. (BOSS Collaboration), Mon. Not. R. Astron. Soc. 470, 2617 (2017).

[3] D. J. Eisenstein, H.-j. Seo, E. Sirko, and D. Spergel, Astrophys. J. 664, 675 (2007).

[4] N. Padmanabhan, M. White, and J. D. Cohn, Phys. Rev. D 79, 063523 (2009).

[5] M. Schmittfull, Y. Feng, F. Beutler, B. Sherwin, and M. Y. Chu, Phys. Rev. D 92, 123522 (2015).

[6] M. Schmittfull, T. Baldauf, and M. Zaldarriaga, Phys. Rev. D 96, 023505 (2017).

[7] I. Esteban, M. C. Gonzalez-Garcia, A. HernandezCabezudo, M. Maltoni, and T. Schwetz, J. High Energy Phys. 01 (2019) 106.

[8] N. Palanque-Delabrouille et al., J. Cosmol. Astropart. Phys. 11 (2015) 011.

[9] A. J. Cuesta, V. Niro, and L. Verde, Phys. Dark Universe 13, 77 (2016).

[10] A. Upadhye, J. Cosmol. Astropart. Phys. 05 (2019) 041.

[11] C. Doux, M. Penna-Lima, S. D. P. Vitenti, J. Tréguer, E. Aubourg, and K. Ganga, Mon. Not. R. Astron. Soc. 480, 5386 (2018).

[12] S. Vagnozzi, E. Giusarma, O. Mena, K. Freese, M. Gerbino, S. Ho, and M. Lattanzi, Phys. Rev. D 96, 123503 (2017).

[13] N. Palanque-Delabrouille, C. Yèche, N. Schöneberg, J. Lesgourgues, M. Walther, S. Chabanier, and E. Armengaud, arXiv:1911.09073.

[14] S. R. Choudhury and S. Hannestad, arXiv:1907.12598.

[15] M. Aker et al. (KATRIN Collaboration), Phys. Rev. Lett. 123, 221802 (2019).

[16] M. M. Ivanov, M. Simonovic, and M. Zaldarriaga, arXiv: 1909.05277.
[17] G. D’Amico, J. Gleyzes, N. Kokron, D. Markovic, L. Senatore, P. Zhang, F. Beutler, and H. Gil-Marín, arXiv: 1909.05271.

[18] T. Colas, G. D'amico, L. Senatore, P. Zhang, and F. Beutler, arXiv:1909.07951.

[19] T. Tröster et al., Astron. Astrophys. 633, L10 (2020).

[20] F. Beutler et al. (BOSS Collaboration), Mon. Not. R. Astron. Soc. 466, 2242 (2017).

[21] K. N. Abazajian et al., arXiv:1204.5379.

[22] M. Drewes et al., J. Cosmol. Astropart. Phys. 01 (2017) 025 .

[23] D. Green et al., Bull. Am. Astron. Soc. 51, 159 (2019), https://arxiv.org/abs/1903.04763.

[24] D. Baumann, D. Green, and B. Wallisch, J. Cosmol. Astropart. Phys. 08 (2018) 029.

[25] D. Baumann, F. Beutler, R. Flauger, D. Green, M. VargasMagaña, A. Slosar, B. Wallisch, and C. Yèche, Nat. Phys. 15, 465 (2019).

[26] H. Gil-Marĩn, L. Verde, J. Noreña, A. J. Cuesta, L. Samushia, W. J. Percival, C. Wagner, M. Manera, and D. P. Schneider, Mon. Not. R. Astron. Soc. 452, 1914 (2015).

[27] F. Beutler et al. (BOSS Collaboration), Mon. Not. R. Astron. Soc. 444, 3501 (2014).

[28] J. N. Grieb et al. (BOSS Collaboration), Mon. Not. R. Astron. Soc. 467, 2085 (2017).

[29] A. G. Sanchez et al. (BOSS Collaboration), Mon. Not. R. Astron. Soc. 464, 1640 (2017).

[30] M. Pellejero-Ibanez et al. (BOSS Collaboration), Mon. Not. R. Astron. Soc. 468, 4116 (2017).

[31] L. Amendola et al., Living Rev. Relativity 21, 2 (2018).

[32] A. Aghamousa et al. (DESI Collaboration), arXiv:1611 .00036 .

[33] D. Baumann, A. Nicolis, L. Senatore, and M. Zaldarriaga, J. Cosmol. Astropart. Phys. 07 (2012) 051. 
[34] J. J. M. Carrasco, M. P. Hertzberg, and L. Senatore, J. High Energy Phys. 09 (2012) 082.

[35] L. Senatore and M. Zaldarriaga, arXiv:1409.1225.

[36] L. Senatore, J. Cosmol. Astropart. Phys. 11 (2015) 007.

[37] A. Perko, L. Senatore, E. Jennings, and R. H. Wechsler, arXiv:1610.09321.

[38] A. Chudaykin and M. M. Ivanov, arXiv:1907.06666.

[39] L. Senatore and M. Zaldarriaga, J. Cosmol. Astropart. Phys. 02 (2015) 013.

[40] Z. Vlah, M. White, and A. Aviles, J. Cosmol. Astropart. Phys. 09 (2015) 014.

[41] Z. Vlah, U. Seljak, M. Y. Chu, and Y. Feng, J. Cosmol. Astropart. Phys. 03 (2016) 057.

[42] T. Baldauf, M. Mirbabayi, M. Simonović, and M.Zaldarriaga, Phys. Rev. D 92, 043514 (2015).

[43] D. Blas, M. Garny, M. M. Ivanov, and S. Sibiryakov, J. Cosmol. Astropart. Phys. 07 (2016) 052.

[44] D. Blas, M. Garny, M. M. Ivanov, and S. Sibiryakov, J. Cosmol. Astropart. Phys. 07 (2016) 028.

[45] M. M. Ivanov and S. Sibiryakov, J. Cosmol. Astropart. Phys. 07 (2018) 053.

[46] S. Pueblas and R. Scoccimarro, Phys. Rev. D 80, 043504 (2009).

[47] M. Lewandowski, A. Perko, and L. Senatore, J. Cosmol. Astropart. Phys. 05 (2015) 019.

[48] V. Desjacques, D. Jeong, and F. Schmidt, Phys. Rep. 733, 1 (2018).

[49] J. C. Jackson, Mon. Not. R. Astron. Soc. 156, 1 P (1972).

[50] J. Lesgourgues and S. Pastor, Phys. Rep. 429, 307 (2006).

[51] E. Di Valentino et al. (CORE Collaboration), J. Cosmol. Astropart. Phys. 04 (2018) 017.

[52] T. Brinckmann and J. Lesgourgues, Phys. Rev. D 97, 063506 (2018).

[53] N. Aghanim et al. (Planck Collaboration), arXiv:1907 .12875 .

[54] H. d. M. des Bourboux et al., Astron. Astrophys. 608, A130 (2017).

[55] M. Ata et al., Mon. Not. R. Astron. Soc. 473, 4773 (2018).

[56] Y. Feng, U. Seljak, and M. Zaldarriaga, J. Cosmol. Astropart. Phys. 07 (2018) 043.

[57] C. Modi, Y. Feng, and U. Seljak, J. Cosmol. Astropart. Phys. 10 (2018) 028.

[58] M. Schmittfull, M. Simonović, V. Assassi, and M. Zaldarriaga, Phys. Rev. D 100, 043514 (2019).

[59] F. Schmidt, F. Elsner, J. Jasche, N. M. Nguyen, and G. Lavaux, J. Cosmol. Astropart. Phys. 01 (2019) 042.

[60] F. Elsner, F. Schmidt, J. Jasche, G. Lavaux, and N.-M. Nguyen, J. Cosmol. Astropart. Phys. 01 (2020) 029.

[61] C. Hahn, R. Scoccimarro, M. R. Blanton, J. L. Tinker, and S. A. Rodríguez-Torres, Mon. Not. R. Astron. Soc. 467, 1940 (2017).

[62] A. Nicola, A. Refregier, and A. Amara, Phys. Rev. D 94, 083517 (2016).

[63] D. Blas, M. Garny, T. Konstandin, and J. Lesgourgues, J. Cosmol. Astropart. Phys. 11 (2014) 039.
[64] L. Senatore and M. Zaldarriaga, arXiv:1707.04698.

[65] M. Takada, E. Komatsu, and T. Futamase, Phys. Rev. D 73, 083520 (2006).

[66] F. Villaescusa-Navarro, F. Marulli, M. Viel, E. Branchini, E. Castorina, E. Sefusatti, and S. Saito, J. Cosmol. Astropart. Phys. 03 (2014) 011.

[67] E. Castorina, E. Sefusatti, R. K. Sheth, F. VillaescusaNavarro, and M. Viel, J. Cosmol. Astropart. Phys. 02 (2014) 049.

[68] M. Costanzi, F. Villaescusa-Navarro, M. Viel, J.-Q. Xia, S. Borgani, E. Castorina, and E. Sefusatti, J. Cosmol. Astropart. Phys. 12 (2013) 012.

[69] E. Castorina, C. Carbone, J. Bel, E. Sefusatti, and K. Dolag, J. Cosmol. Astropart. Phys. 07 (2015) 043.

[70] F. Villaescusa-Navarro, A. Banerjee, N. Dalal, E. Castorina, R. Scoccimarro, R. Angulo, and D. N. Spergel, Astrophys. J. 861, 53 (2018).

[71] A. Raccanelli, L. Verde, and F. Villaescusa-Navarro, Mon. Not. R. Astron. Soc. 483, 734 (2019).

[72] S. Vagnozzi, T. Brinckmann, M. Archidiacono, K. Freese, M. Gerbino, J. Lesgourgues, and T. Sprenger, J. Cosmol. Astropart. Phys. 09 (2018) 001.

[73] N. Kaiser, Mon. Not. R. Astron. Soc. 227, 1 (1987).

[74] C.-T. Chiang, W. Hu, Y. Li, and M. Loverde, Phys. Rev. D 97, 123526 (2018).

[75] C.-T. Chiang, M. LoVerde, and F. Villaescusa-Navarro, Phys. Rev. Lett. 122, 041302 (2019).

[76] J. Hamann, S. Hannestad, J. Lesgourgues, C. Rampf, and Y. Y. Y. Wong, J. Cosmol. Astropart. Phys. 07 (2010) 022.

[77] F. Beutler et al. (BOSS Collaboration), Mon. Not. R. Astron. Soc. 464, 3409 (2017).

[78] V. Yankelevich and C. Porciani, Mon. Not. R. Astron. Soc. 483, 2078 (2019).

[79] B. Audren, J. Lesgourgues, S. Bird, M. G. Haehnelt, and M. Viel, J. Cosmol. Astropart. Phys. 01 (2013) 026.

[80] A. Font-Ribera, P. McDonald, N. Mostek, B. A. Reid, H.-J. Seo, and A. Slosar, J. Cosmol. Astropart. Phys. 05 (2014) 023.

[81] M. Archidiacono, T. Brinckmann, J. Lesgourgues, and V. Poulin, J. Cosmol. Astropart. Phys. 02 (2017) 052.

[82] T. Sprenger, M. Archidiacono, T. Brinckmann, S. Clesse, and J. Lesgourgues, J. Cosmol. Astropart. Phys. 02 (2019) 047.

[83] T. Brinckmann, D. C. Hooper, M. Archidiacono, J. Lesgourgues, and T. Sprenger, J. Cosmol. Astropart. Phys. 01 (2019) 059.

[84] D. Blas, J. Lesgourgues, and T. Tram, J. Cosmol. Astropart. Phys. 07 (2011) 034.

[85] B. Audren, J. Lesgourgues, K. Benabed, and S. Prunet, J. Cosmol. Astropart. Phys. 02 (2013) 001.

[86] M. Simonovic, T. Baldauf, M. Zaldarriaga, J. J. Carrasco, and J. A. Kollmeier, J. Cosmol. Astropart. Phys. 04 (2018) 030 .

[87] A. Lewis and S. Bridle, Phys. Rev. D 66, 103511 (2002).

[88] A. Lewis, Phys. Rev. D 87, 103529 (2013). 